\title{
Mucormycosis: Current Update in Pandemic COVID-19
}

\author{
Anish Khan ${ }^{1 *}$, Aishwarya Soni' ${ }^{2}$ and Amit Kumar ${ }^{3}$ \\ ${ }^{1}$ Centre for Biotechnology, Maharshi Dayanand University, Rohtak-124001, \\ Haryana, India \\ ${ }^{2}$ Department of Biotechnology, Deenbandhu Chhotu Ram University of Science and \\ Technology-Murthal, Sonepat-131039, Haryana, India \\ ${ }^{3}$ Assistant Professor, Head of the Department of Zoology, Government College for \\ Women, Gohana-131301, Sonepat, Haryana, India \\ *Corresponding Author: Anish Khan, Centre for Biotechnology, Maharshi Day- \\ anand University, India. Email: anishkhan9991@gmail.com
}

DOI: 10.31080/ASMI.2021.04.0883

\section{Background}

Corona virus disease-2019 (COVID-19) is a pandemic entity caused by a novel corona virus (severe acute respiratory syndrome-coronavirus-2; SARS-CoV-2), which was firstly reported in Wuhan, Hubei territory in China on December 2019 [1,2]. SARSCoV-2 is continuing to spread worldwide to till date with a high proportion of infected or affecting $\geq 10$ million individuals globally [1]. Until now, there is no permanent cure for pandemic COVID-19 disease, though a variety of executive options have been evaluated and managed with systemic glucocorticoids for recovery of endurance rate of COVID-19 patients [2]. However, an array of complications reported during and post COVID-19 infection due to extensive use of glucocorticoids, which may lead to secondary fungal co-infection $[1,2]$.

Recently, Garg., et al. [2] study a case report on COVID-19 associated with mucormycosis (MM) usually developed in 10-14 days after hospitalization and higher risk in diabetes mellitus, chronic obstructive pulmonary disease and immunocompromised patients. MM (formally known as Zygomycosis) is extremely scattering to immunocompromised patients, who have uncontrolled diabetic conditions. However, MM is an angioinvasive infection after Aspergillus and Candida spp. in humans, which is acute, extremely opportunist and ubiquitous belonging to class Zygomycetes, i.e. 'pin molds' (formerly known as Phygomycetes). Zygomycetes quickly colonizes on nutriment rich carbohydrates medium like glucose as main energy source for its survival. Moreover, its spore's spreads via oral, nasal and conjunctival mucosa by inhalation or ingestion of contaminated food materials [3]. Normally, MM is a non/sparsely septate fungus, which grow most often if individual have weaken immunity and resulting a high mortality rates exceeding of $60 \%$ with promising complications such as partial loss of neurological functions, blindness, clotting of brain or lung vessels, etc. $[3,4]$.

Jestin., et al. [5] found to be a high incidence rate of 7.3\% (per year) for MM infection during period of 2001 to 2010. Prevalence rate of MM is 0.14 cases per 1000 individual in developed countries, while its rate increase 80 times in developing countries like India [4]. Similarly, Millon., et al. [6] observed a prevalence and fatality rate of $7 \%$ and $9.3 \%$ in the United States and France, respectively during period 2000-2010. Nevertheless, Furbinger (1876) firstly investigated a patient who died with cancer and in whom right lung showed a hemorrhagic infarct with few sporangia of MM. The European Confederation of Medical Mycology and International Society of Human and Animal Mycology groups working on MM to constructed a registry in 2004 (www.zygomyco.net) due to high mortality rate spreads by MM.

Several approaches have been developed, i.e. clinical signs/ symptoms, microscopic viewing (wet mount, calcofluor white, periodic acid-schiff stain, gomori methenamine silver stain, FISH, immunohistochemistry), mycological cultures (API-ID50C/ID32C), serology, radiology, histopathology, etc. that can confirm diagnosis of invasive pin molds infection but these assays elusive, lack 
variable sensitivities and specificities [7-9]. Aggressive nature of MM that delayed in diagnosis and treatment thus use of molecular approaches such as conventional PCR targeting mucorales $18 \mathrm{~S}$ ribosomal DNA, spore coating protein homolog encoding genes (uniquely/universally present among all mucorales), RFLP, DNA sequencing, Real-time PCR (RT-PCR), matrix assisted laser desorption/ionization time-of-flight mass spectrometry (MALDI-TOF/ MS) have been good utility for providing species identity of mucorales [8,10]. Moreover, serological tests including MALDI-TOF/ MS, metabolomics and metagenomic shotgun sequencing are other advanced technologies may be helpful for MM diagnosis. The editorial editorial note is to develop new molecular methods for mucormycetes spp. identification and proper treatment. Currently, most of the physician cum-researcher recommended a liposomal amphotericin-B with posaconazole and isavuconazole drugs are main choice for treatment of MM [9].

\section{Conclusion}

Over the past decade, a number of published studies on MM infection increased and reflects growing problems in highly immunocompromised patients. So, we need to improve for early diagnosis and understanding therapeutic management of MM by molecular methods. Moreover, diagnosis of MM could be possible using antigens/antibodies or DNA/RNA-based selection tools. Overall, we should focus on standardization/optimization of molecular techniques and their evaluation of merits incorporating into routine diagnostic algorithms.

\section{Acknowledgements}

A Khan and A Kumar acknowledge to Ms. Deepika (AS Cancer Biology) for providing an opportunities for editorials note.

\section{Conflicts of Interest}

The authors declare that there are no conflicts of interest.

\section{Bibliography}

1. Sarkar S., et al. "COVID-19 and orbital mucormycosis". Indian Journal of Ophthalmology 69.4 (2021): 1002.

2. Garg D., et al. "Coronavirus disease (Covid-19) associated mucormycosis (CAM): case report and systematic review of literature". Mycopathologia (2021): 1-10
3. Prabhu RM and Robin P. "Mucormycosis and entomophthoramycosis: a review of the clinical manifestations, diagnosis and treatment". Clinical Microbiology and Infection 10 (2004): 3147.

4. Skiada A., et al. "Challenges in the diagnosis and treatment of mucormycosis”. Medical Mycology 56 (2018): S93-S101.

5. Jestin M., et al. "Poor outcome associated with mucormycosis in critically ill hematological patients: results of a multicenter study". Annals of Intensive Care 11.1 (2021): 1-8.

6. Millon L., et al. "Molecular strategies to diagnose mucormycosis". Journal of Fungi 5.1 (2019): 24.

7. Dadwal SS and Dimitrios PK. "Recent advances in the molecular diagnosis of mucormycosis". Expert review of Molecular Diagnostics 18.10 (2018): 845-854.

8. Jeong W., et al. "The epidemiology and clinical manifestations of mucormycosis: a systematic review and meta-analysis of case reports". Clinical Microbiology and Infection 25.1 (2019): 26-34.

9. Lackner M., et al. "Laboratory diagnosis of mucormycosis: current status and future perspectives". Future Microbiology 9.5 (2014): 683-695.

10. Kidd SE, et al. "A new age in molecular diagnostics for invasive fungal disease: are we ready?". Frontiers in Microbiology 10 (2020): 2903.

\section{Volume 4 Issue 8 August 2021}

\section{(C) All rights are reserved by Anish Khan., et al.}

